hep-th/0212225

\title{
Adding Fundamental Matter to "Chiral Rings and Anomalies in Supersymmetric Gauge Theory"
}

\author{
Nathan Seiberg \\ School of Natural Sciences, Institute for Advanced Study, Princeton NJ 08540 USA
}

\begin{abstract}
We consider a supersymmetric $U(N)$ gauge theory with matter fields in the adjoint, fundamental and anti-fundamental representations. As in the framework which was put forward by Dijkgraaf and Vafa, this theory can be described by a matrix model. We analyze this theory along the lines of [F. Cachazo, M. Douglas, N.S. and E. Witten, "Chiral Rings and Anomalies in Supersymmetric Gauge Theory" hep-th/0211170 and show the equivalence of the gauge theory and the matrix model. In particular, the anomaly equations in the gauge theory is identified with the loop equations in the matrix model.
\end{abstract}

December 2002 


\section{Introduction}

Recently Dijkgraaf and Vafa [1] were motivated by earlier work [2-8] to conjecture an interesting relation between SUSY gauge theories and matrix models. Many authors have added matter in the fundamental representation [9 25] to this framework. We will examine a general theory of this form which includes the various examples of [9-25] as special cases. We will follow the point of view of [26], and will extend it to this case with fundamental matter.

We consider an $\mathcal{N}=1$ supersymmetric $U(N)$ gauge theory with matter in the adjoint

$\Phi, N_{f}$ fundamentals $Q^{f}$ and $N_{f}$ anti-fundamentals $\widetilde{Q}_{\widetilde{f}}(f$ and $\widetilde{f}$ are the flavor indices). The tree level superpotential is

$$
W_{\text {tree }}=\operatorname{Tr} W(\Phi)+\widetilde{Q}_{\tilde{f}} m_{f}^{\widetilde{f}}(\Phi) Q^{f}
$$

where we suppressed the color indices. The function $W$ and the matrix $m$ are taken to be polynomials

$$
\begin{aligned}
W(z) & =\sum_{k=1}^{n} \frac{1}{k+1} g_{k} z^{k+1} \\
m_{f}^{\widetilde{f}}(z) & =\sum_{k=1}^{l+1} m_{f, k}^{\widetilde{f}} z^{k-1}
\end{aligned}
$$

We consider arbitrary $N$ and $N_{f}$. For $n N_{f}>N$ these theories exhibit a certain duality 2729] exchanging $N \leftrightarrow n N_{f}-N$, and we hope that this discussion will shed light on it.

In section 2 we analyze this quantum field theory focusing on its chiral ring, the anomaly equations and the low energy effective superpotential. In section 3 we consider the corresponding matrix model and prove its equivalence to the gauge theory.

\section{Gauge theory considerations}

\subsection{Anomaly equations}

As in [26] we will be interested in the chiral operators

$$
\begin{aligned}
T(z) & =\operatorname{Tr} \frac{1}{z-\Phi} \\
w_{\alpha}(z) & =\frac{1}{4 \pi} \operatorname{Tr} \frac{W_{\alpha}}{z-\Phi} \\
R(z) & =-\frac{1}{32 \pi^{2}} \operatorname{Tr} \frac{W_{\alpha} W^{\alpha}}{z-\Phi} \\
M_{\widetilde{f}}^{f}(z) & =\widetilde{Q}_{\widetilde{f}} \frac{1}{z-\Phi} Q^{f}
\end{aligned}
$$


Since $W_{\alpha} Q^{f}$ and $\widetilde{Q}_{\widetilde{f}} W_{\alpha}$ are not in the chiral ring, there is no need to include more operators 1 . Also, since the gauge group is $U(N)$ rather than $S U(N)$ we do not include "baryonic operators."

We perform the following five independent transformations in the functional integral

$$
\begin{aligned}
\delta \Phi & =\frac{1}{z-\Phi} \\
\delta \Phi & =\frac{W_{\alpha}}{z-\Phi} \\
\delta \Phi & =\frac{W_{\alpha} W^{\alpha}}{z-\Phi} \\
\delta Q^{f} & =\frac{1}{z-\Phi} \lambda_{f^{\prime}}^{f} Q^{f^{\prime}} \\
\delta \widetilde{Q}_{\widetilde{f}} & =\widetilde{\lambda}_{\widetilde{f}}^{\widetilde{f}^{\prime}} \widetilde{Q}_{\widetilde{f}^{\prime}} \frac{1}{z-\Phi}
\end{aligned}
$$

where $\lambda_{f^{\prime}}^{f}$ and $\widetilde{\lambda}_{\widetilde{f}}^{\widetilde{f}^{\prime}}$ are constant flavor matrices. The anomaly equations of these transformations are

$$
\begin{aligned}
& \operatorname{Tr} \frac{W^{\prime}(\Phi)}{z-\Phi}+\widetilde{Q}_{\widetilde{f}} \frac{m_{f}^{\prime \widetilde{f}}(\Phi)}{z-\Phi} Q^{f}=2 R(z) T(z)+w_{\alpha}(z) w^{\alpha}(z) \\
& \frac{1}{4 \pi} \operatorname{Tr} \frac{W^{\prime}(\Phi) W_{\alpha}}{z-\Phi}=2 R(z) w_{\alpha}(z) \\
& -\frac{1}{32 \pi^{2}} \operatorname{Tr} \frac{W^{\prime}(\Phi) W_{\alpha} W^{\alpha}}{z-\Phi}=R(z)^{2} \\
& \lambda_{f^{\prime}}^{f} \widetilde{Q}_{\widetilde{f}} \frac{m_{f}^{\widetilde{f}}(\Phi)}{z-\Phi} Q^{f^{\prime}}=\lambda_{f}^{f} R(z) \\
& \widetilde{\lambda}_{\widetilde{f}}^{\widetilde{f}^{\prime}} \widetilde{Q}_{\widetilde{f}^{\prime}} \frac{m_{f}^{\widetilde{f}}(\Phi)}{z-\Phi} Q^{f}=\widetilde{\lambda}_{\widetilde{f}}^{\widetilde{f}} R(z)
\end{aligned}
$$

where the equalities are only in the chiral ring; i.e. they are up to SUSY commutators. The $\mathcal{O}(1 / z)$ term in the first equation is the equation of motion of $\operatorname{Tr} \Phi$. The $\mathcal{O}\left(1 / z^{2}\right)$ term in that equation and the $\mathcal{O}(1 / z)$ terms in the fourth and fifth equations are the Konishi anomaly [30,31] for $\Phi, Q$ and $\widetilde{Q}$ respectively. The other equations and the other powers of $1 / z$ are various generalizations.

1 We thank E. Witten for a useful discussion on this point. 
Since $W(\Phi)$ and $m_{f}^{\widetilde{f}}(\Phi)$ are polynomials, we can replace these equations with

$$
\begin{aligned}
& {\left[W^{\prime}(z) T(z)\right]_{-}+\operatorname{tr}\left[m^{\prime}(z) M(z)\right]_{-}=2 R(z) T(z)+w_{\alpha}(z) w^{\alpha}(z)} \\
& {\left[W^{\prime}(z) w_{\alpha}(z)\right]_{-}=2 R(z) w_{\alpha}(z)} \\
& {\left[W^{\prime}(z) R(z)\right]_{-}=R(z)^{2}} \\
& {\left[(M(z) m(z))_{f}^{f^{\prime}}\right]_{-}=R(z) \delta_{f}^{f^{\prime}}} \\
& {\left[(m(z) M(z))_{\widetilde{f}}^{\widetilde{f^{\prime}}}\right]_{-}=R(z) \delta_{\widetilde{f}}^{\widetilde{f^{\prime}}}}
\end{aligned}
$$

Here $m(z)$ and $M(z)$ are matrices in flavor space. We multiply such matrices as $(A B)_{f^{\prime \prime}}^{f}=$ $A_{f^{\prime}}^{f} B_{f^{\prime \prime}}^{f^{\prime}}$ and $\operatorname{tr}$ denotes a trace over the flavor indices. In the last two equations we used the fact that the corresponding equations in (2.3) are satisfied for every $\lambda$ and $\widetilde{\lambda}$. These identities are also true for the expectation values of the operators (2.1).

Writing the third equation in (2.4) as

$$
W^{\prime}(z) R(z)+\frac{1}{4} f(z)=R(z)^{2}
$$

with a polynomial $f(z)=-4\left[W^{\prime}(z) R(z)\right]_{+}$of degree $n-1$, we solve for $R(z)$

$$
2 R(z)=W^{\prime}(z)-\sqrt{W^{\prime}(z)^{2}+f(z)}
$$

Similarly, we write the last two equations in (2.4) as

$$
\begin{aligned}
& (M(z) m(z)-q(z))_{f}^{f^{\prime}}=R(z) \delta_{f}^{f^{\prime}} \\
& (m(z) M(z)-\widetilde{q}(z)){\widetilde{f^{\prime}}}_{\widetilde{f}}=R(z) \delta_{\widetilde{f}}^{\widetilde{f}^{\prime}}
\end{aligned}
$$

in terms of polynomial matrices

$$
\begin{aligned}
& q(z)=[M(z) m(z)]_{+} \\
& \widetilde{q}(z)=[m(z) M(z)]_{+}
\end{aligned}
$$

We can now solve for the matrix $M(z)$

$$
M(z)=R(z) m^{-1}(z)+q(z) m^{-1}(z)=R(z) m^{-1}(z)-m^{-1}(z) \widetilde{q}(z)
$$

where $m^{-1}(z)$ is the inverse matrix. For consistency the polynomial matrices $q(z)$ and $\widetilde{q}(z)$ are related by $\widetilde{q}=m q m^{-1}$. Using the solution for $R(z)$ in (2.6) the expression for $M(z)$ (2.9) leads to a solution for $M(z)$ in terms of the polynomials $f(z)$ and $q(z)$. 
The polynomial $f(z)$ is kept arbitrary, but the polynomials $q(z)$ are fixed as follows. From (2.9) it is clear that $M(z)$ is singular when $m(z)$ has a zero eigenvalue. The freedom in the polynomials $q(z)$ is exactly such as to remove these singularities. To see this, consider for simplicity the case $N_{f}=1$, where the flavor matrices are one dimensional. $m(z)$ is a polynomial of degree $l$ (1.2), and therefore it has $l$ zeroes $z_{I}$. For large $z$ the resolvent $M(z)$ behaves as $\frac{1}{z}$ and therefore $q(z)=[M(z) m(z)]_{+}$is a polynomial of degree $l-1$. The $l$ coefficients of $q$ can be tuned to set the $l$ residues of $M(z)$ at $z_{I}$ to zero.

Now that we have solved for $R(z)$ and $M(z)$, it is simple to solve for $w_{\alpha}(z)$ and $T(z)$ using the first two equations in (2.4). In doing that we need to introduce new polynomials

$$
\begin{aligned}
\rho_{\alpha}(z) & =-4\left[W^{\prime}(z) w_{\alpha}(z)\right]_{+} \\
c(z) & =-4\left[W^{\prime}(z) T(z)\right]_{+}
\end{aligned}
$$

Note that the solution for $R(z)$ is as in the theory without the fundamental matter, but $T(z)$ is different; it depends on $m(z)$.

\subsection{The effective superpotential}

We parametrize the superpotential of the adjoint field as

$$
W^{\prime}(z)=g_{n} \prod_{i=1}^{n}\left(z-a_{i}\right)
$$

in terms of its stationary points $a_{i}$. We consider the classical vacuum

$$
\langle\Phi\rangle=\left(\begin{array}{ccccccccc}
a_{1} & & & & & & & & \\
& \cdot & & & & & & & \\
& & \cdot & & & & & & \\
& & a_{2} & & & & & \\
& & & \cdot & & & & \\
& & & & \cdot & & & \\
& & & & a_{3} & & \\
& & & & & \cdot & & \\
& & & & & & \cdot & \\
& & & & & & & a_{n}
\end{array}\right)
$$

where $a_{i}$ occurs $N_{i}$ times. We assume that $W(\Phi)$ and $m(\Phi)$ are generic and in particular $m\left(a_{i}\right)$ does not have a zero eigenvalue. Since all the matter fields acquire masses, the low 
energy degrees of freedom are only the gauge fields in $\prod_{i} U\left(N_{i}\right)$. Following [26] we express them as the gauge invariant objects

$$
\begin{aligned}
S_{i} & =\frac{1}{2 \pi i} \oint_{C_{i}} R(z) d z \\
w_{\alpha}^{(i)} & =\frac{1}{2 \pi i} \oint_{C_{i}} w_{\alpha}(z) d z \\
N_{i} & =\frac{1}{2 \pi i} \oint_{C_{i}} T(z) d z
\end{aligned}
$$

where $C_{i}$ is a contour around $a_{i}$.

Since we assumed that $m$ is generic, all the quarks are massive and are integrated out. Therefore, our effective Lagrangian will not depend on the "meson operator in the $i$ group"

$$
M^{(i)}=\frac{1}{2 \pi i} \oint_{C_{i}} M(z) d z
$$

As in [26] (see also [32]) we integrate out the massive fields in perturbation theory. It is straightforward to add the fundamental matter to the discussion of the Feynman diagrams in [26]. Assume for simplicity that the group $U(N)$ is unbroken; i.e. $N_{1}=N$. Then considerations of symmetry and holomorphy along the lines of [33] constrain the perturbative effective superpotential to be of the form

$$
W_{e f f}^{p e r t}=W_{\alpha}^{2} F\left(\frac{g_{k} W_{\alpha}^{k-1}}{g_{1}^{\frac{k+1}{2}}}, \frac{m_{f, k}^{\widetilde{f}} W_{\alpha}^{k-1}}{\left(m_{f, k=1}^{\widetilde{f}}\right)^{\frac{k+1}{2}}}\right)
$$

Since the theory depends on several masses $g_{1}, m_{f, k=1}^{\widetilde{f}}$, the function $F$ can also depend on various ratios of them. From (2.15) we see that the number of $W_{\alpha}$ in a diagram that contributes to $W_{\text {eff }}^{\text {pert }}$ is constrained to be

$$
\# W_{\alpha}=2+\sum_{r}\left(k_{r}-1\right)
$$

Here $r$ labels the vertices of the Feynman diagram, and $k_{r}$ is the index $k$ either for $g_{k}$ or for $m_{f, k}^{\widetilde{f}}$ at the vertex. We now write the diagram using 'tHooft's index loops notation. Every $\Phi$ has a double line and every $Q$ or $\widetilde{Q}$ has a single line. The number of index loops in the diagram is

$$
L=2+\frac{1}{2} \sum_{r}\left(k_{r}-1\right)-2 h-b
$$


where $h$ is the number of handles in the surface and $b$ is the number of boundaries $(b$ is the number of $Q$ or $\widetilde{Q}$ index loops). From (2.16) and (2.17)

$$
\# W_{\alpha}=2 L-2+4 h+2 b
$$

As in [26], it is straightforward to extend this discussion to the case where the gauge group is broken, and the external light fields in the diagram are $W_{\alpha}^{(i)}$. Here we find

$$
\sum_{i} \# W_{\alpha}^{(i)}=2 L-2+4 h+2 b
$$

We also know that chiral operators have at most two factors of $W_{\alpha}^{(i)}$ in a trace and therefore there are at most two $W_{\alpha}^{(i)}$ on each index loop:

$$
\sum_{i} \# W_{\alpha}^{(i)} \leq 2 L
$$

Combining (2.19) and (2.20) we learn that only three kinds of diagrams can contribute to the effective superpotential:

1. $h=b=0$. These diagrams have the topology of a sphere. One index loop has no operator insertion (leading to a factor of $N_{i}$ ) and every other index loop has an insertion of $S_{j}$ leading to a result proportional to $N_{i} S_{j}^{L-1}\left(S_{j}^{L-1}\right.$ stands for a product of $L-1$ factors of $S_{j}$ not necessarily all with the same value of $j$ ).

2. $h=b=0$. These diagrams also have the topology of a sphere. Two index loops have an insertion of $w_{\alpha}^{(i)}$ on each, and every other index loop has $S_{j}$ leading to a result proportional to $w_{\alpha}^{(i)} w^{(k) \alpha} S_{j}^{L-2}$.

3. $h=0, b=1$. These diagrams have the topology of a disk. They have an insertion of $S_{j}$ on each index loop leading to a result proportional to $S_{j}^{L}$.

The sphere diagrams do not involve $Q$ and $\widetilde{Q}$. Therefore, their contribution is the same as in the theory without these fields; i.e. having only the adjoint field $\Phi$. This theory has a shift symmetry associated with the decoupled diagonal $U(1)$ field $\sum_{i} w_{\alpha}^{(i)}$ [26]. This symmetry is best implemented by adding the auxiliary spinor coordinate $\psi_{\alpha}$, and combining $S_{i}, w_{\alpha}^{(i)}$ and $N_{i}$ to a superfield

$$
\mathcal{S}_{i}(\psi)=S_{i}+\psi^{\alpha} w_{\alpha}^{(i)}-\psi^{1} \psi^{2} N_{i}
$$

Then the symmetry of shifting $\psi^{\alpha}$ determines the sphere contribution in terms of a single function $\mathcal{F}_{0}^{\text {pert }}$ of $\mathcal{S}_{i}(\psi)$

$$
\int d^{2} \psi \mathcal{F}_{0}^{p e r t}\left(\mathcal{S}_{i}(\psi)\right)
$$


The integral over $\psi$ leads to the two sphere contributions we mentioned above. The function $\mathcal{F}_{0}^{\text {pert }}$ is exactly the same as this function in the theory without the fundamental matter. It is given by

$$
\mathcal{F}_{0}^{\text {pert }}=\sum_{i} \frac{1}{2} \mathcal{S}_{i}^{2} \log \left(\frac{g_{n} \prod_{j \neq i}\left(a_{i}-a_{j}\right)}{\Lambda_{0}}\right)-\sum_{\substack{i, j \\ i \neq j}} \mathcal{S}_{i} \mathcal{S}_{j} \log \frac{a_{i}-a_{j}}{\Lambda_{0}}+\mathcal{O}\left(S_{j}^{3}\right)
$$

Here we wrote explicitly the one loop result which depends on the UV cutoff $\Lambda_{0}$. The first term arises from integrating out the massive chiral superfield in the adjoint of $U\left(N_{i}\right)$, and the second term is from the massive vector superfields which acquired mass in the Higgs mechanism. The higher order terms $\mathcal{O}\left(S_{j}^{3}\right)$ are independent of $\Lambda_{0}$.

The disk diagrams lead to another function of $S_{i}$ whose contribution to the effective superpotential is not integrated over $\psi$

$$
\mathcal{F}_{1}^{p e r t}=\sum_{i} S_{i} \log \left(\operatorname{det} \frac{m\left(a_{i}\right)}{\Lambda_{0}}\right)+\mathcal{O}\left(S_{j}^{2}\right)
$$

where we wrote explicitly the one loop terms which arise from the massive quarks $Q$ and $\widetilde{Q}$. Again, the higher order terms $\mathcal{O}\left(S_{j}^{2}\right)$ are independent of the UV cutoff $\Lambda_{0}$. $\mathcal{F}_{1}^{\text {pert }}$ is not integrated over $\psi$ and it does not respect the shift symmetry of $\psi$. This is consistent with the fact that in the theory with fundamental matter the over all $U(1)$ superfield $\sum_{i} w_{\alpha}^{(i)}$ does not decouple.

The full effective superpotential at the scale $\mu$ where the gauge dynamics of the unbroken $\prod_{i} U\left(N_{i}\right)$ gauge fields is still weak is

$$
W_{\text {eff }}^{\text {pert }}=\tau_{0} \sum_{i} S_{i}+\frac{1}{2} \log \left(\frac{\Lambda_{0}}{\mu}\right)^{3} \sum_{i} \int d^{2} \psi \mathcal{S}_{i}^{2}(\psi)+\int d^{2} \psi \mathcal{F}_{0}^{\text {pert }}\left(\mathcal{S}_{i}(\psi)\right)+\mathcal{F}_{1}^{\text {pert }}\left(S_{i}\right)
$$

The first term is the bare coupling. The second term includes the one loop running of the gauge couplings in $\prod_{i} S U\left(N_{i}\right)$; it depends only on the $S U\left(N_{i}\right)$ fields $s_{i}=S_{i}+\frac{1}{2 N_{i}}\left(w_{\alpha}^{(i)}\right)^{2}$

$$
\frac{1}{2} \log \left(\frac{\Lambda_{0}}{\mu}\right)^{3} \sum_{i} \int d^{2} \psi \mathcal{S}_{i}^{2}(\psi)=\sum_{i} N_{i} s_{i} \log \left(\frac{\Lambda_{0}}{\mu}\right)^{3}
$$

The dependence on the UV cutoff $\Lambda_{0}$ from all the terms is

$$
\left(2 N-N_{f}\right) \log \Lambda_{0} \sum_{i} S_{i}+\log \Lambda_{0}\left(\sum_{i} w_{\alpha}^{(i)}\right)^{2}
$$


In accordance with the one loop beta function we choose the bare coupling

$$
\tau_{0}=-\left(2 N-N_{f}\right) \log \Lambda_{0} / \Lambda
$$

with a finite scale $\Lambda$. This has the effect of changing $\Lambda_{0} \rightarrow \Lambda$ everywhere except the term $\log \Lambda_{0}\left(\sum_{i} w_{\alpha}^{(i)}\right)^{2}$. This indicates that the over all $U(1)$ superfield $\sum_{i} w_{\alpha}^{(i)}$ is free in the renormalized theory. We will continue to use the same notation as in (2.23)-(2.26) but with $\Lambda_{0} \rightarrow \Lambda$.

The strong IR dynamics is implemented by replacing (2.26) (after $\Lambda_{0} \rightarrow \Lambda$ ) with the Veneziano-Yankielowics superpotential [34]

$$
W_{V Y}\left(s_{i}\right)=\sum_{i} s_{i}\left(\log \frac{\Lambda^{3 N_{i}}}{s_{i}^{N_{i}}}+N_{i}\right)=\frac{1}{2} \sum_{i} \int d^{2} \psi \mathcal{S}_{i}^{2}\left(\log \frac{\Lambda^{3}}{\mathcal{S}_{i}}+\frac{3}{2}\right)
$$

Therefore, the final answer for the effective superpotential is

$$
W_{\text {eff }}\left(S_{i}, w_{\alpha}^{(i)}, N_{i}\right)=\int d^{2} \psi \mathcal{F}_{0}\left(\mathcal{S}_{i}(\psi)\right)+\mathcal{F}_{1}\left(S_{i}\right)=\int d^{2} \psi\left(\mathcal{F}_{0}\left(\mathcal{S}_{i}(\psi)\right)+\psi^{2} \psi^{1} \mathcal{F}_{1}\left(\mathcal{S}_{i}(\psi)\right)\right)
$$

with

$$
\begin{aligned}
& \mathcal{F}_{0}=\mathcal{F}_{0}^{\text {pert }}+\frac{1}{2} \sum_{i} \mathcal{S}_{i}^{2}\left(\log \frac{\Lambda^{3}}{\mathcal{S}_{i}}+\frac{3}{2}\right) \\
& \mathcal{F}_{1}=\mathcal{F}_{1}^{\text {pert }}
\end{aligned}
$$

Here $s_{i}=S_{i}+\frac{1}{2 N_{i}}\left(w_{\alpha}^{(i)}\right)^{2}$ are independent chiral superfields, and $w_{\alpha}^{(i)}$ are independent field strengths of the massless vector superfields.

Using the effective superpotential we can compute expectation values of operators by differentiating with respect to the coefficients in (11.2)

$$
\begin{aligned}
& \frac{1}{k+1}\left\langle\operatorname{Tr} \Phi^{k+1}\right\rangle=\frac{\partial W_{\text {eff }}}{\partial g_{k}}=\frac{\partial}{\partial g_{k}} \int d^{2} \psi \mathcal{F}_{0}+\frac{\partial \mathcal{F}_{1}}{\partial g_{k}} \\
& \left\langle\widetilde{Q}_{\widetilde{f}} \Phi^{k-1} Q^{f}\right\rangle=\frac{\partial W_{\text {eff }}}{\partial m_{f, k}^{\widetilde{f}}}=\frac{\partial \mathcal{F}_{1}}{\partial m_{f, k}^{\widetilde{f}}}
\end{aligned}
$$

In the second equation we used the fact that the sphere diagrams do not involve the fundamental matter, and therefore the function $\mathcal{F}_{0}$ is independent of $m_{f, k}^{\widetilde{f}}$. The sphere contribution in the first equation is as in the theory without the fundamental matter. The disk contribution depends on $m_{f, k}^{\widetilde{f}}$. Therefore $\left\langle\operatorname{Tr} \Phi^{k+1}\right\rangle$ and $\langle T(z)\rangle$ depend on $m_{f, k}^{\widetilde{f}}$ in accord with the comment after (2.10). 
Since we have already solved for these expectation values using the anomaly equations, we effectively found the full effective superpotential. The variables $S_{i}$ or more precisely $s_{i}$ capture the information in the coefficients in the polynomial $f(z)$ in (2.5). Equivalently, the freedom in $f(z)$ is determined by the values of the fields $s_{i}$. The polynomials $\rho_{\alpha}(2.10)$ are determined in terms of the variables $w_{\alpha}^{(i)}$. Finally, $c(z)(2.10)$ is determined in terms of $N_{i}$. We remarked above that $M^{(i)}$ (2.14) are integrated out. This is closely related to the fact that the polynomials $q(z)$ (2.8) are determined (see the discussion after (2.9)). An ambiguity in $q(z)$ would have appeared as more fields in the effective superpotential.

\section{Matrix model}

We consider the matrix model with the "action"

$$
A=\frac{\widehat{N}}{\widehat{g}}\left[\operatorname{Tr} W(\widehat{\Phi})+\widehat{\widetilde{Q}}_{\widetilde{f}_{f}} m_{f}^{\tilde{f}}(\widehat{\Phi}) \widehat{Q}^{f}\right]
$$

where $\widehat{\Phi}, \widehat{\widetilde{Q}}$ and $\widehat{Q}$ are $\widehat{N} \times \widehat{N}, \widehat{N} \times N_{f}$ and $N_{f} \times \widehat{N}$ dimensional matrices, and define its free energy $\widehat{\mathcal{F}}$ through

$$
\exp \left(-\frac{\widehat{N}^{2}}{\widehat{g}^{2}} \widehat{\mathcal{F}}\right)=\int d \widehat{\Phi} d \widehat{Q} d \widehat{\widetilde{Q}} \exp (-A)
$$

Eventually, we will take the large $\widehat{N}$ limit with everything else held fixed.

We will be interested in the resolvents

$$
\begin{array}{r}
\widehat{R}(z)=\frac{\widehat{g}}{\widehat{N}}\left\langle\operatorname{Tr} \frac{1}{z-\widehat{\Phi}}\right\rangle \\
\widehat{M}_{\widetilde{f}}^{f}(z)=\left\langle\widehat{\widetilde{Q}}_{\widetilde{f}} \frac{1}{z-\widehat{\Phi}^{f}} \widehat{Q}^{f}\right\rangle
\end{array}
$$

We perform the following three independent transformations

$$
\begin{aligned}
\delta \widehat{\Phi} & =\frac{1}{z-\widehat{\Phi}} \\
\delta \widehat{Q}^{f} & =\frac{1}{z-\widehat{\Phi}_{f^{\prime}}} \lambda^{f} \widehat{Q}^{f^{\prime}} \\
\delta \widehat{\widetilde{Q}}_{\widetilde{f}} & =\widehat{\widetilde{Q}}_{\widetilde{f}^{\prime}} \widetilde{\lambda}_{\widetilde{f}}^{\widetilde{f}^{\prime}} \frac{1}{z-\widehat{\Phi}}
\end{aligned}
$$


to find

$$
\begin{aligned}
& \frac{\widehat{N}}{\widehat{g}}\left[\operatorname{Tr} \frac{W^{\prime}(\widehat{\Phi})}{z-\widehat{\Phi}}+\widehat{\widetilde{Q}} \frac{m^{\prime}(\widehat{\Phi})}{z-\widehat{\Phi}} \widehat{Q}\right]=\left(\operatorname{Tr} \frac{1}{z-\widehat{\Phi}}\right)^{2} \\
& \frac{\widehat{N}}{\widehat{g}} \widehat{\widetilde{Q}} \frac{m(\widehat{\Phi})}{z-\widehat{\Phi}} \lambda \widehat{Q}=\operatorname{tr} \lambda \operatorname{Tr} \frac{1}{z-\widehat{\Phi}} \\
& \frac{\widehat{N}}{\widehat{\widetilde{Q}}} \widetilde{\lambda} \frac{m(\widehat{\Phi})}{z-\widehat{\Phi}} \widehat{Q}=\operatorname{tr} \widetilde{\lambda} \operatorname{Tr} \frac{1}{z-\widehat{\Phi}}
\end{aligned}
$$

where we suppressed both the matrix indices and the flavor indices. Again we used the convention $A_{f}^{f^{\prime}} B_{f^{\prime \prime}}^{f}=(A B)_{f^{\prime \prime}}^{f^{\prime}}$, and tr denotes a trace over the flavor indices. Taking the expectation values of these equations and the large $\widehat{N}$ limit they become

$$
\begin{aligned}
& {\left[W^{\prime}(z) \widehat{R}(z)\right]_{-}=\widehat{R}(z)^{2}} \\
& {\left[(\widehat{M}(z) m(z))_{f}^{f^{\prime}}\right]_{-}=\widehat{R}(z) \delta_{f}^{f^{\prime}}} \\
& {\left[(m(z) \widehat{M}(z))_{\widetilde{f}}^{\widetilde{f^{\prime}}}\right]_{-}=\widehat{R}(z) \delta_{\widetilde{f}}^{\widetilde{f^{\prime}}}}
\end{aligned}
$$

In the last two equations we used the fact that they are true for every $\lambda$ and $\tilde{\lambda}$.

The equations (3.6) are the loop equation [35] of the matrix model. The first equation describes a closed string splitting to two closed strings. The other two equations describe an open string turning into a closed string.

In solving the first equation for $\widehat{R}(z)$ there is an ambiguity in a polynomial $\widehat{f}(z)=$ $-4\left[W^{\prime}(z) \widehat{R}(z)\right]_{+}$. However, in solving for $\widehat{M}(z)$ the ambiguity in $\widehat{q}(z)=[m(z) \widehat{M}(z)]_{+}$is fixed by imposing that $\widehat{M}(z)$ is not singular at the points where $m(z)$ has a zero eigenvalue.

The equations (3.6) are the same as the last three equations in the gauge theory (2.4) and the ambiguity in solving them (the value of $\widehat{f}(z)$ and the way $\widehat{q}(z)$ is determined) is also the same. Therefore we can identify

$$
\begin{aligned}
\widehat{R}(z) & =\langle R(z)\rangle \\
\widehat{M}(z) & =\langle M(z)\rangle
\end{aligned}
$$

It is interesting that while $\left\langle\operatorname{Tr} \frac{1}{z-\widehat{\Phi}}\right\rangle$ in the matrix model is not identified with $\left\langle\operatorname{Tr} \frac{1}{z-\Phi}\right\rangle$ in the gauge theory but with $\left\langle\operatorname{Tr} \frac{W_{\alpha}^{2}}{z-\Phi}\right\rangle$, the matrix model object $\left\langle\widehat{\widetilde{Q}} \frac{1}{z-\widehat{\Phi}} \widehat{Q}\right\rangle$ is identified with its natural gauge theory counterpart $\left\langle\widetilde{Q} \frac{1}{z-\Phi} Q\right\rangle$.

We now relate the effective superpotential in the gauge theory 2.30 to the free energy of the matrix model $\widehat{\mathcal{F}}$. We will need the sphere and the disk contributions to the free 
energy: $\widehat{\mathcal{F}}_{0}=\lim _{\widehat{N} \rightarrow \infty} \widehat{\mathcal{F}}, \widehat{\mathcal{F}}_{1}=\lim _{\widehat{N} \rightarrow \infty} \frac{\widehat{N}}{\widehat{g}}\left(\widehat{\mathcal{F}}-\widehat{\mathcal{F}}_{0}\right) . \widehat{\mathcal{F}}_{0}$ is computed in the theory without the fundamental matter. It was conjectured by Dijkgraaf and Vafa [1], and proven in [26] (see also [32]) that in this theory $\mathcal{F}_{0}=\widehat{\mathcal{F}}_{0}$ (recall that $\mathcal{F}_{0}$ in the gauge theory (2.22) (2.30) is the same as in the theory without the fundamental matter). We now turn to the disk amplitudes. Following (3.7)

$$
\left\langle\widetilde{Q}_{\widetilde{f}^{-}} \Phi^{k-1} Q^{f}\right\rangle=\left\langle\widehat{\widetilde{Q}}_{\widetilde{f}} \widehat{\Phi}^{k-1} \widehat{Q}^{f}\right\rangle
$$

They can be computed in the matrix model and in the gauge theory as derivatives with respect to the coefficients in $m_{f}^{\widetilde{f}}(z)$

$$
\frac{\partial \mathcal{F}_{1}}{\partial m_{f, k}^{\widetilde{f}}}=\frac{\partial \widehat{\mathcal{F}}_{1}}{\partial m_{f, k}^{\widetilde{f}}}
$$

where we used (2.32) and the fact that in the matrix model $\widehat{\mathcal{F}}_{0}$ is independent of $m_{f, k} \widetilde{f}$. We learn that $\mathcal{F}_{1}=\widehat{\mathcal{F}}_{1}+\delta \mathcal{F}_{1}$ with $\delta \mathcal{F}_{1}$ independent of $m_{f, k}^{\widetilde{f}}$. Using the special case with only $m_{f, k=1}^{\widetilde{f}}=m \delta_{f}^{\widetilde{f}}$ nonzero and $m \rightarrow \infty$ we easily learn that $\delta \mathcal{F}_{1}=0$.

We conclude that

$$
\begin{aligned}
\mathcal{F}_{0} & =\widehat{\mathcal{F}}_{0} \\
\mathcal{F}_{1} & =\widehat{\mathcal{F}}_{1}
\end{aligned}
$$

This establishes the equivalence of the gauge theory and the matrix model for this case.

\section{Acknowledgements}

It is a pleasure to thank D. Berenstein, F. Cachazo, M. Douglas, D. Kutasov, G. Moore and E. Witten for helpful discussions. This work was supported in part by DOE grant \#DE-FG02-90ER40542 to IAS. 


\section{References}

[1] R. Dijkgraaf and C. Vafa, "A perturbative window into non-perturbative physics," arXiv:hep-th/0208048.

[2] M. Bershadsky, S. Cecotti, H. Ooguri and C. Vafa, "Kodaira-Spencer theory of gravity and exact results for quantum string amplitudes," Commun. Math. Phys. 165, 311 (1994) arXiv:hep-th/9309140.

[3] R. Gopakumar and C. Vafa, "On the gauge theory/geometry correspondence," Adv. Theor. Math. Phys. 3, 1415 (1999) arXiv:hep-th/9811131.

[4] C. Vafa, "Superstrings and topological strings at large N," J. Math. Phys. 42, 2798 (2001) arXiv:hep-th/0008142.

[5] F. Cachazo, K. A. Intriligator and C. Vafa, "A large N duality via a geometric transition," Nucl. Phys. B 603, 3 (2001) arXiv:hep-th/0103067.

[6] F. Cachazo and C. Vafa, "N = 1 and $\mathrm{N}=2$ geometry from fluxes," arXiv:hepth/0206017.

[7] R. Dijkgraaf and C. Vafa, "Matrix models, topological strings, and supersymmetric gauge theories," arXiv:hep-th/0206255.

[8] R. Dijkgraaf and C. Vafa, "On geometry and matrix models," arXiv:hep-th/0207106.

[9] T. J. Hollowood and T. Kingaby, "The phase structure of mass-deformed $\mathrm{SU}(2) \mathrm{x}$ $\mathrm{SU}(2)$ quiver theory," arXiv:hep-th/0210096.

[10] D. Berenstein, "Quantum moduli spaces from matrix models," arXiv:hep-th/0210183.

[11] R. Argurio, V. L. Campos, G. Ferretti and R. Heise, "Exact superpotentials for theories with flavors via a matrix integral," arXiv:hep-th/0210291.

[12] J. McGreevy, "Adding flavor to Dijkgraaf-Vafa," arXiv:hep-th/0211009.

[13] H. Suzuki, "Perturbative derivation of exact superpotential for meson fields from matrix theories with one flavour," arXiv:hep-th/0211052.

[14] I. Bena and R. Roiban, "Exact superpotentials in $\mathrm{N}=1$ theories with flavor and their matrix model formulation," arXiv:hep-th/0211075.

[15] Y. Demasure and R. A. Janik, "Effective matter superpotentials from Wishart random matrices," arXiv:hep-th/0211082.

[16] Y. Tachikawa, "Derivation of the Konishi anomaly relation from Dijkgraaf-Vafa with (bi-)fundamental matters," arXiv:hep-th/0211189.

[17] R. Argurio, V. L. Campos, G. Ferretti and R. Heise, "Baryonic corrections to superpotentials from perturbation theory," arXiv:hep-th/0211249.

[18] S. G. Naculich, H. J. Schnitzer and N. Wyllard, "Matrix model approach to the N $=2 \mathrm{U}(\mathrm{N})$ gauge theory with matter in the fundamental representation," arXiv:hepth/0211254.

[19] I. Bena, R. Roiban and R. Tatar, "Baryons, boundaries and matrix models," arXiv:hep-th/0211271. 
[20] B. Feng, "Seiberg duality in matrix model," arXiv:hep-th/0211202.

[21] B. Feng and Y. H. He, "Seiberg duality in matrix models. II," arXiv:hep-th/0211234.

[22] Y. Ookouchi, "N = 1 gauge theory with flavor from fluxes," arXiv:hep-th/0211287.

[23] K. Ohta, "Exact mesonic vacua from matrix models," arXiv:hep-th/0212025.

[24] I. Bena, S. de Haro and R. Roiban, "Generalized Yukawa couplings and matrix models," arXiv:hep-th/0212083.

[25] C. Hofman, "Super Yang-Mills with flavors from large N(f) matrix models," arXiv:hepth/0212095.

[26] F. Cachazo, M. R. Douglas, N. Seiberg and E. Witten, "Chiral rings and anomalies in supersymmetric gauge theory," arXiv:hep-th/0211170.

[27] D. Kutasov, "A Comment on duality in $\mathrm{N}=1$ supersymmetric nonAbelian gauge theories," Phys. Lett. B 351, 230 (1995) arXiv:hep-th/9503086.

[28] D. Kutasov and A. Schwimmer, "On duality in supersymmetric Yang-Mills theory," Phys. Lett. B 354, 315 (1995) arXiv:hep-th/9505004.

[29] D. Kutasov, A. Schwimmer and N. Seiberg, "Chiral Rings, Singularity Theory and Electric-Magnetic Duality," Nucl. Phys. B 459, 455 (1996) [arXiv:hep-th/9510222.

[30] K. Konishi, "Anomalous Supersymmetry Transformation Of Some Composite Operators In Sqcd," Phys. Lett. B 135, 439 (1984).

[31] K. i. Konishi and K. i. Shizuya, "Functional Integral Approach To Chiral Anomalies In Supersymmetric Gauge Theories," Nuovo Cim. A 90, 111 (1985).

[32] R. Dijkgraaf, M. T. Grisaru, C. S. Lam, C. Vafa and D. Zanon, "Perturbative Computation of Glueball Superpotentials," arXiv:hep-th/0211017.

[33] N. Seiberg, "Naturalness versus supersymmetric nonrenormalization theorems," Phys. Lett. B 318, 469 (1993) arXiv:hep-ph/9309335.

[34] G. Veneziano and S. Yankielowicz, "An Effective Lagrangian For The Pure N=1 Supersymmetric Yang-Mills Theory," Phys. Lett. B 113, 231 (1982).

[35] A. A. Migdal, "Loop Equations And 1/N Expansion," Phys. Rept. 102, 199 (1983). 